\title{
A Study of Hybridization and Architectural Effects on Tensile Response of 3D Woven Textile Composites using the NCYL Multiscale Method
}

\author{
Deepak K. Patel* and Anthony M. Waas ${ }^{\dagger}$ \\ Aerospace Engineering, University of Michigan, Ann Arbor, MI, 48109 \\ William E Boeing Department of Aeronautics and Astronautics, University of Washington, Seattle, WA 98195
}

\begin{abstract}
This paper presents results of numerical simulations on predicting the progressive damage and failure response of hybrid 3D woven textile composites (3DWTC) subjected to tensile loading, using a novel two-scale computational mechanics framework. Here the term 'hybrid' refers to different constituent fibers, including carbon, glass and kevlar that are infused with SC-15 polymer matrix and integrally woven into a single preform. The hybrid 3DWTCs are made through a 3D textile weaving process. Three different versions of hybridized architectures are examined at the unit cell level to determine the progression of damage and failure under tensile loading. The pre-peak nonlinearity, as caused by matrix microdamage is modeled using the N-layer concentric cylinder model (NCYL) ${ }^{1,2}$ and the post-peak softening failure response is modeled using a mesh-objective smeared crack approach (SCA $)^{3,4}$ in a two-scale framework. A micro-CT analysis of all three architectures is conducted and FE models are generated directly from Micro-CT data using the software tool 'Simpleware'. These FE models are the real replication of in-situ geometric imperfections in the structure and subsequently used for progressive damage and failure analysis using the two-scale method. A detailed explanation of the method for a hybrid 2.5D woven textile composite is provided in Patel and Waas. ${ }^{5}$ The three different types of hybrid 3DWTCs are compared against one another to understand the benefits of hybridization and the resulting performance enhancements. The numerical simulation results show the effect of microstructure geometric imperfections on the prediction of progressive damage and failure response and are also verified against experimental results. ${ }^{6}$
\end{abstract}

\section{Introduction}

Three dimensional woven textile composites (3DWTC) are recognised as a potential high performance composite material for applications in aeroapsce, civil and other industry sectors. 3DWTC possess a great advantage of high resistance to delamination, as the latter is a common mode of failure in conventional laminated composites. The mechanical performance can be enhanced due to reinforcement in the thickness direction and the in-plane properties are significantly improved due to attempts at aligning the in-plane fiber tows. This unique signature is a characteristic of the 3D textile weaving process but at significantly reduced manufacturing cost and time, compared to traditional pre-preg based materials. The design methods adopted for 3DWTC structures are still based on testing and a reliable computational predictive model is indispensible. Such a model should be based on the physics of material response and failure, using constitutive model properties that can be measured independently through a set of coupon level tests.

${ }^{*}$ PhD Candidate, Aerospace Engineering, University of Michigan, Ann Arbor, MI, 48109

${ }^{\dagger}$ Boeing-Egtvedt Chair, Professor of Aerostructures, Chair, William E Boeing Department of Aeronautics and Astronautics , University of Washington, Seattle, WA 98195. Fellow AIAA

Copyright (C)2017 by Deepak K. Patel and Anthony M. Waas. Published with permission by the American Institute of Aeronautics and Astronautics. 
However, the hierarchical nature of composites makes the characterization and modeling of such materials a great challenge. Most of the damage and failure events evolve at the constituent material scale with little knowledge on the in-situ constituent properties. Predictions from computational models, therefore, may encompass a high level of uncertainty. For large-scale structural analysis, homogenization at the macroscale is essential to achieve computational efficiency, whereas the model also needs to consider the influence of the underlying microstructure.

Multiscale modeling is a suitable and efficient methodology for progressive damage and failure analysis of 3DWTC, where information is shared across different length scales. The proposed computational scheme should be able to bridge the gap between micro and macro scale in a cost effective way. In the literature, a number of micromechanics models have been developed, as reviewed in. ${ }^{7,8}$ Zhang and Waas ${ }^{1}$ proposed a micromechanics-based two-scale model which is able to predict the nonlinear response of unidirectional composites. Their proposed micromechanics analysis uses the analytical solutions derived from the concentric cylinder model along with the generalized self-consistent model to find the effective properties of the unidirectional fiber reinforced composite and also the stress and strain fields within the fiber and matrix. The composite material is represented by an inner fiber core and an outer matrix annulus. Recently, Patel, Hasanyan and $\mathrm{Waas}^{2}$ extended the 2-CYL fiber-matrix concentric cylinder model into a concentric fiber and any number of (N-1) matrix layers in general, keeping the volume fraction constant, referred to as the N-cylinder micromechanics model (NCYL), which can analytically calculate the strain and stress field within the fiber and matrix cylinders for a given applied remote composite strain field, acting on the outer boundary of the matrix cylinder. The spatial variation of strain and stress fields within the cylinder are the key determinants for progressive damage and failure analysis of composite structures and hence, these fields need to be predicted with high accuracy. The advantage of the NCYL model is that the matrix strain and stress fields can be found in a discrete manner for each layer and the evolution of damage can be localized at a particular layer of the matrix. These results are used at the sub-scale in a 2-scale analysis to calculate the effective nonlinear composite response at the macro-scale. Because the sub-scale computation relies on an analytical solution, a distinct computational advantage in composite progressive damage and failure analysis is evident, compared against a fully computational model at each scale.

Three dimensional, orthogonal woven composites are a class of 3DWTC composite structures containing a set of fiber tows spreading in all three mutually perpendicular directions ( $\mathrm{x}, \mathrm{y}$ and $\mathrm{z}$-axes). 3DWTC is manufactured by laying up the warp and weft fiber layers and consequently running a Z-fiber in the thickness direction to bind the in-plane layers together. A matrix material is impregnated into these complex woven lay-ups and cured under certain condition to get the woven solid structure. ${ }^{6}$ The material system (fiber and matrix materials) used in manufacturing these hybrid complex architectures are detailed in Section II. Although, there are many forms of 3D woven composite with the variations of Z-fiber depth in thickness direction, we will focus here mainly on three different configurations, which are described in Section III. The purpose of this paper is to establish a multiscale computational framework to predict the progressive damage and failure response of these complex 3D woven textile composites using constitutive properties measured from coupon level tests. The microstructure characterization studies are provided in Section IV. Details of the modeling strategy, including the construction of the finite element (FE) model, are presented in Section V. The determination of the input properties for the 2CYL model and the development of the matrix nonlinear constitutive model are provided in Section VI. Various fiber and matrix failure modes are included in this study and their implementation in the smeared crack approach (SCA) are explained in Section VII. The predictive capability of the proposed method is evaluated by comparing the computational results of various textile configurations and loading conditions with experiment, as discussed in Section VIII, followed by conclusions in Section IX.

\section{Material system}

This paper discusses a hybrid Z-fiber orthogonal interlock textile architecture, in which three different types of fibers, IM-7 carbon, S-2 glass and Kevlar are integrally woven into a single preform. These hybrid architectures are provided by Textile Enginnering and Manufacturing (T.E.A.M.), Inc., RI. The hybridization of the composite material can be tailored for the specific need of the structure by varying the fiber type and configuration of the weaving layers. The carbon fiber is used because of their high strength and stiffness, the glass for its relatively low cost (high strength per unit cost) and kevlar for its high resistance (characterized by its toughness) to failure. Material properties for each fiber type and the matrix are provided in Table 1. 
Table 1. The elastic properties for IM-7 carbon fiber, S-2 glass fiber, kevlar fiber and SC-15 matrix.

\begin{tabular}{cccccc}
\hline & $E_{1}$ & $E_{2}$ & $\nu_{12}$ & $G_{12}$ & $G_{23}$ \\
& $\mathrm{GPa}$ & $\mathrm{GPa}$ & - & $\mathrm{GPa}$ & $\mathrm{GPa}$ \\
\hline IM7 Carbon Fiber & 276.0 & 15.0 & 0.279 & 12.0 & 5.02 \\
S-2 Glass Fiber & 93.8 & & 0.23 & 38.1 & \\
Kevlar Fiber & 112 & & 0.36 & 41.2 & \\
SC-15 Matrix & 2.487 & & 0.35 & 0.921 & \\
\hline
\end{tabular}

\section{Textile Architecture}

The warp and weft fibers run in-plane throughout the panel with little or no undulation. A set of Z-fibers run in the direction of warp fibers and are drawn from bottom to the top to bind all the layers together. Z-fibers are usually inserted in-between the spaces of the warp fibers. In this paper, three different hybrid architectures, as schematically shown in Figure 1 are investigated to understand the effect of hybridization on the resulting tensile response. The first two architectures contain four layers of carbon (two layers in the warp and weft directions, respectively) at one side, and the remainder are the glass layers. These hybrid configurations are unsymmetric architecture that consists of orthogonally oriented in-plane carbon and the glass tows, for a total 9 layers, named as Thin Asymmetric and of total 17 layers, named as Thick Asymmetric respectively. The difference in the overall thickness allows for studying the size-scaling effects by normalizing the result with respect to the panel thickness. These two architectures are considered to be unsymmetric in the sense that the types of the layered constituent fibers are unsymmetric with respect to the mid-plane of the composite panel. The third architecture contains the carbon layers for both outer surfaces (four layers of carbon on each side) and nine layers of glass in the middle. This hybrid configuration is a symmetric architecture that consists of orthogonally oriented in-plane carbon and glass tows (totaling 17 layers) and named as Thick Symmetric. The Z-fibers run half the thickness in two layers in all Thick configurations to bind the layers together. This is a distinct feature of this architecture (compared to the through thickness binding in the 3D Thin Asymmetric configuration). These types of woven composites are categorized as 2.5D Thick Symmetric/Asymmetric configurations for Z-fibers running half the thickness.

Among all the architectures, the planes that contain Z-fibers alternate along the weft direction with a period of two to achieve a repeating textile pattern. These configurations are infused with SC-15 polymer matrix material using a VARTM process. The three hybrid architectures are further examined under microscopy to identify unit cells and characterize the in-situ imperfections. The geometric dimensions of unit cells and layer breakdown for these architectures are summarized in Table 2.

Table 2. Unit cell dimensions and layer breakdown

\begin{tabular}{ccccccc}
\hline Configuration & $\begin{array}{c}\text { Dimension }(\mathrm{mm}) \\
(\text { WxH } \mathrm{H})\end{array}$ & $\begin{array}{c}\text { Total } \\
\text { Layers }\end{array}$ & $\begin{array}{c}\text { Carbon } \\
\text { Layers }\end{array}$ & $\begin{array}{c}\text { Carbon } \\
(\%)\end{array}$ & $\begin{array}{c}\text { Glass } \\
\text { Layers }\end{array}$ & $\begin{array}{c}\text { Glass } \\
(\%)\end{array}$ \\
\hline Thin Asymmetric & $9.6 \times 8.75 \times 17.2$ & 9 & 4 & 44.4 & 5 & 55.6 \\
Thick Asymmetric & $6.8 \times 16.8 \times 16$ & 17 & 4 & 23.5 & 13 & 76.5 \\
Thick Symmetric & $6.8 \times 15.96 \times 16$ & 17 & 8 & 47.1 & 9 & 52.9 \\
\hline
\end{tabular}

The percentage of the carbon and glass content in each architecture is calculated by dividing the number of carbon and glass layers by the total layers of the composite. The images of the polished surface as shown in Figure 2 demonstrates high straightness of the in-plane tows and high uniformity of the geometry for all configurations. For weft tows, the waviness is highest in certain layers as caused by the Z-fibers, where the kevlar fibers change directions from top to bottom or vice-versa and clearly visible in the figure. The fiber tow waviness and the crookedness are further examined with optical microscopy to characterize the textile architecture and geometric imperfections.

\section{Micro-CT analysis of textile configurations}

In order to obtain a thorough understanding of the microstructure of the cured composite, cross sectional microscopic images are used to characterize the textile geometry. The $2 \mathrm{D}$ images at various cross sections 


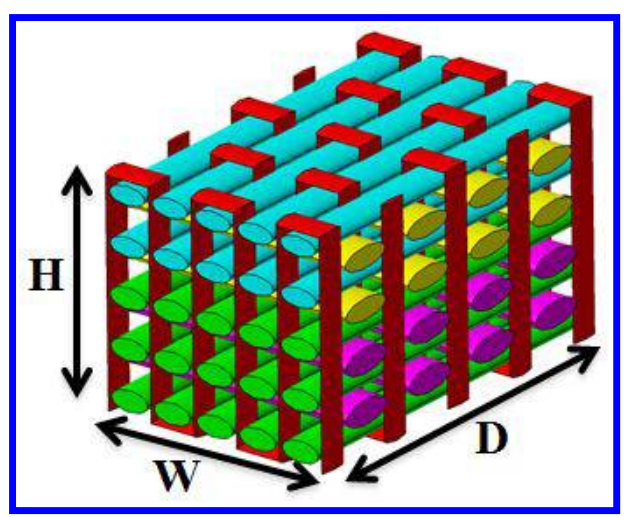

(a) Thin Asymmetric

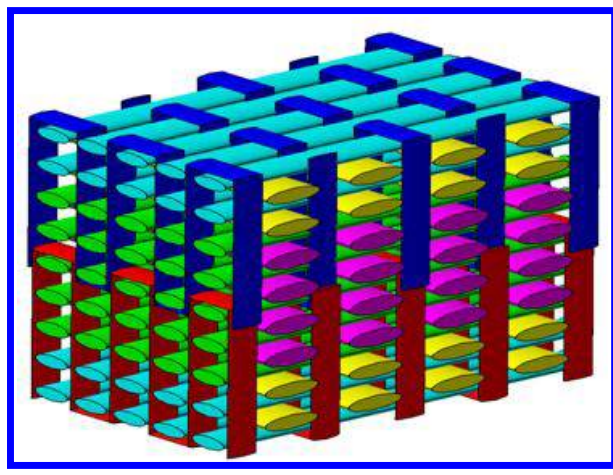

(c) Thick Symmetric

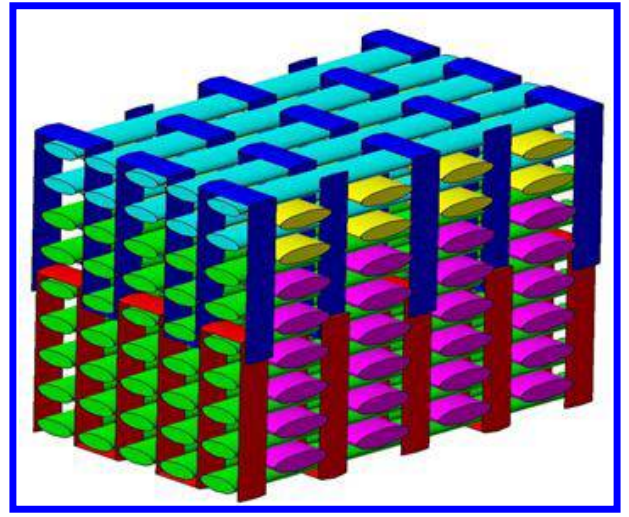

(b) Thick Asymmetric

Figure 1. Hybrid 3D woven textile configurations (Schematic)

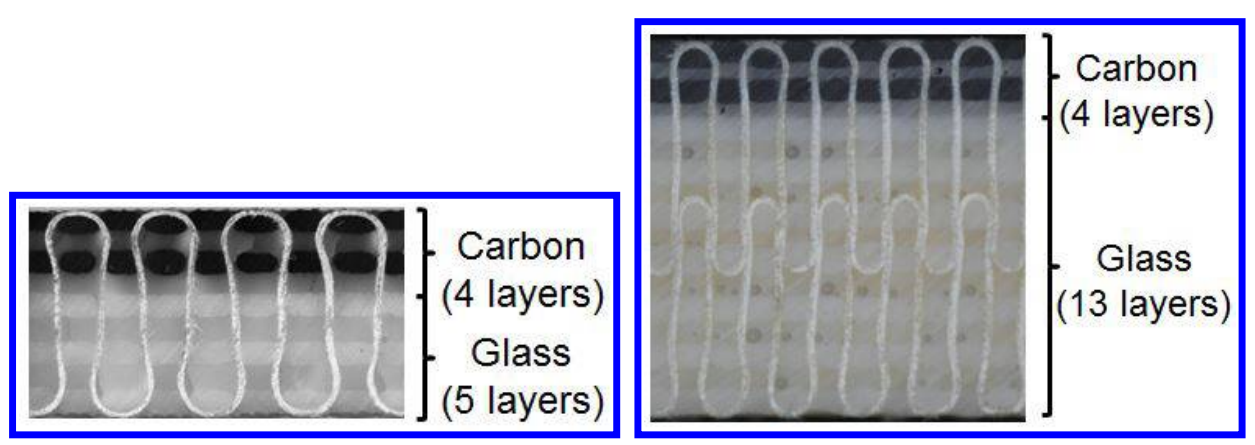

(a) Thin Asymmetric

(b) Thick Asymmetric

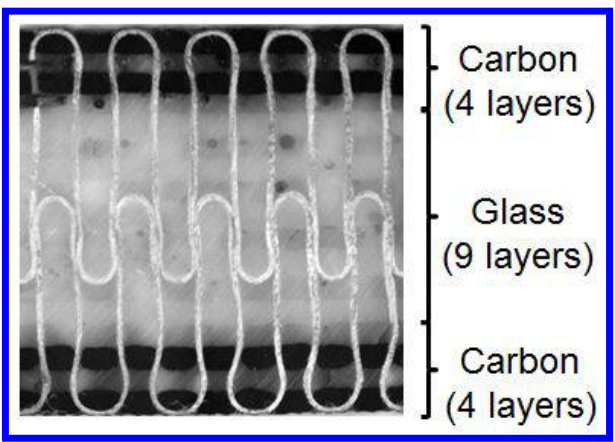

(c) Thick Symmetric

Figure 2. Cross sections parallel to the warp direction (image: Dr Mark Pankow, Dr Dianyun Zhang) 
along the length of the specimen are rendered and reconstructed into 3D volume for the purpose of characterization. The measured dimensions are used as inputs to textile architecture based finite element models presented in Section V. The Micro-CT scanning parameter settings and procedure are detailed in Patel and Waas. $^{5}$

\section{IV.A. Determining unit cell}

A unit cell scanned image data was cropped down from the larger specimen volume data. Cross sections along the mid plane of fiber tows are chosen selectively to capture the periodicity of unit cell in both inplane directions. Unit cell in the black box is indicated in both warp and weft directions for all Thick Asymmetric/Symmetric and Thin Asymmetric configurations, as shown in Figure 3 and summarized in Table 2 .

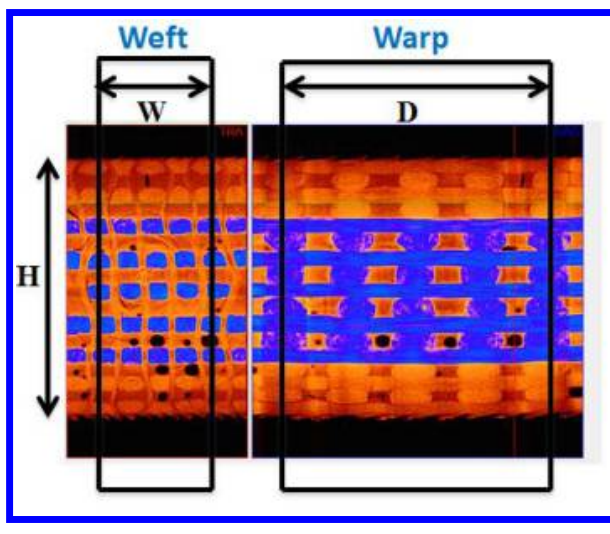

(a) Thick Asymmetric/Symmetric

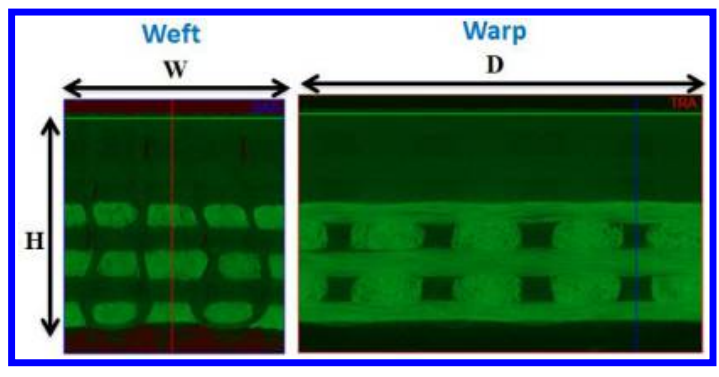

(b) Thin Asymmetric

Figure 3. Micro-CT analysis to determine unit cell

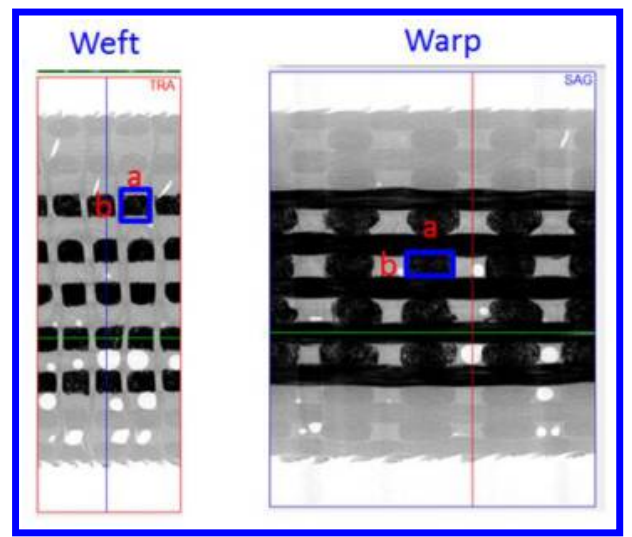

(a) Thick Asymmetric/Symmetric

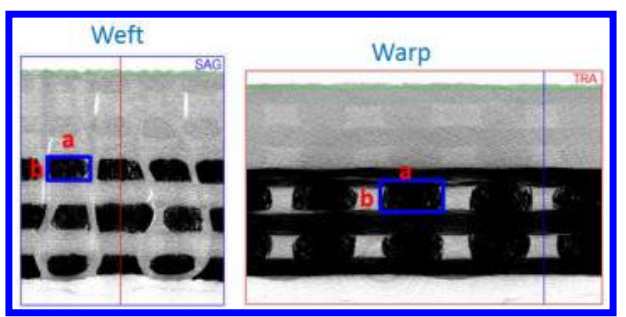

(b) Thin Asymmetric

Figure 4. Measurement of fiber tow details

\section{IV.B. Determining fiber tow cross-sectional details}

Fiber tow cross section dimensions are measured from the unit cell image data using a measuring tool in both warp and weft directions, as indicated in Figure 4. Both the warp and weft tows are assumed to be straight with rectangular cross sections and the measured fiber tow dimensions are summarized in Table 3. SEM images are taken on the cross section of each type of constituent fiber tow to investigate how the fibers are distributed inside the tow and to determine the average fiber volume fraction. The fiber volume fraction of each constituent tow is summarized in Table 4. 
Table 3. Fiber tow cross-section dimensions

\begin{tabular}{ccc}
\hline FiberType & $a(\mathrm{~mm})$ & $b(\mathrm{~mm})$ \\
\hline Weft Carbon & 2.2 & 0.85 \\
Warp Carbon & 2.6 & 0.75 \\
Weft Glass & 2.1 & 1.00 \\
Warp Glass & 2.5 & 0.85 \\
Kevlar & 1.4 & 0.25 \\
\hline
\end{tabular}

Table 4. Volume fraction data for different fiber types

\begin{tabular}{cc}
\hline FiberType & VolumeFraction \\
\hline Carbon & $60.9 \pm 3.6 \%(60 \%)$ \\
Glass & $55.4 \pm 4.5 \%(56 \%)$ \\
Kevlar & $76 \%$ \\
\hline
\end{tabular}

\section{Numerical Model}

An idealized 3D CAD unit cell of the Thin Asymmetric, Thick Asymmetric and Thick Symmetric woven textile composites are modeled in the commercial finite element (FE) software ABAQUS 6.12, as shown in Figure 5 below, taking into account all the input values from Micro-CT study without any geometrical imperfections.

\section{V.A. Modeling in-situ geometry imperfections}

'Simpleware' is a software tool which deals with real scanned image data from Micro-CT analysis. It can generate an FE mesh from Micro-CT real data, which helps in measuring and modeling in-situ imperfections in the structure. Each fiber tow is segmented separately and assigned material properties with well defined fiber orientation as shown in Figure 6. The thin layer of matrix between the undulated fiber tows are meshed explicitly and assigned the material properties of SC-15 epoxy. In this novel and unique approach, modeling and meshing the in-situ geometrical imperfections are possible in a detailed and efficient manner. The measured imperfections can be taken as a reference to introduce imperfections to an idealized perfect geometry. The identified Unit Cells of all three configurations are meshed explicitly using the features of 'Simpleware', as shown in Figure 7. It should be noted here that, the generated mesh is a replication of the in-situ geometric imperfections in the 3DWTC architecture, induced due to the manufacturing process.

\section{Characterization of Constituent Properties}

In this research, the matrix in-situ properties were determined from a tensile test of a $\pm 45^{\circ}$ symmetric laminate in conjunction with a micromechanics model. Details of this method are provided in. ${ }^{9}$ Furthermore, it is assumed that the matrix nonlinear response can be characterized using an exponential relation,

$$
\sigma_{e q}=\sigma_{y}-\frac{K_{1}}{K_{2}}\left(\mathrm{e}^{-K_{2} \epsilon_{e q}}-\mathrm{e}^{-K_{2} \frac{\sigma_{y}}{E}}\right)
$$

where $\sigma_{y}$ is the yield stress of the matrix in a uniaxial tension test, $E$ is the elastic modulus, $K_{1}$ and $K_{2}$ are two material constants that govern the evolution of matrix microdamage. The equivalent stress, $\sigma_{e q}$, and the equivalent strain, $\epsilon_{e q}$, are computed based upon each stress and strain component as,

$$
\begin{aligned}
\sigma_{e q} & =\sqrt{\frac{1}{2}\left[\left(\sigma_{11}-\sigma_{22}\right)^{2}+\left(\sigma_{22}-\sigma_{33}\right)^{2}+\left(\sigma_{33}-\sigma_{11}\right)^{2}\right]+3\left(\sigma_{12}^{2}+\sigma_{13}^{2}+\sigma_{23}^{2}\right)} \\
\epsilon_{e q} & =\frac{1}{1+\nu_{s}} \sqrt{\frac{1}{2}\left[\left(\epsilon_{11}-\epsilon_{22}\right)^{2}+\left(\epsilon_{22}-\epsilon_{33}\right)^{2}+\left(\epsilon_{33}-\epsilon_{11}\right)^{2}\right]+\frac{3}{4}\left(\gamma_{12}^{2}+\gamma_{13}^{2}+\gamma_{23}^{2}\right)}
\end{aligned}
$$

where $\nu_{s}$ is the matrix secant Poisson's ratio defined by,

$$
\nu_{s}=\frac{1}{2}+\frac{E_{s}}{E}\left(\nu-\frac{1}{2}\right)
$$




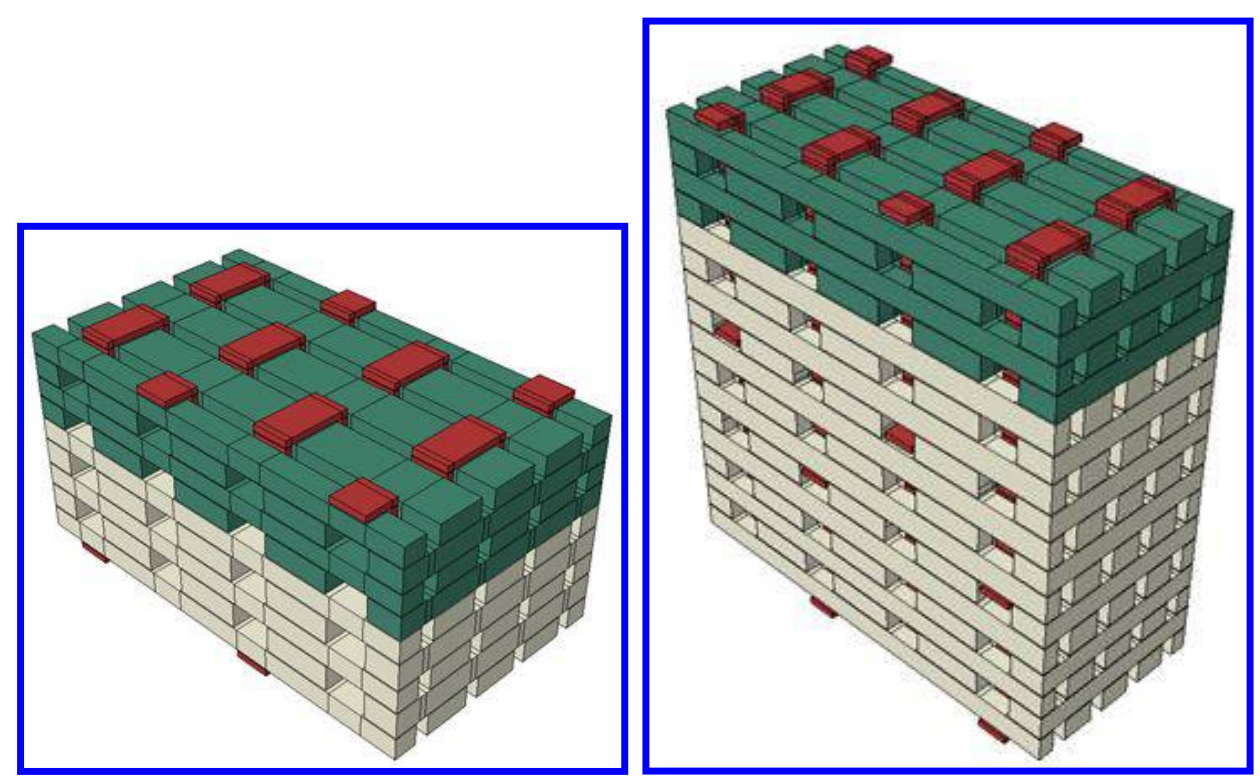

(a) Thin Asymmetric

(b) Thick Asymmetric

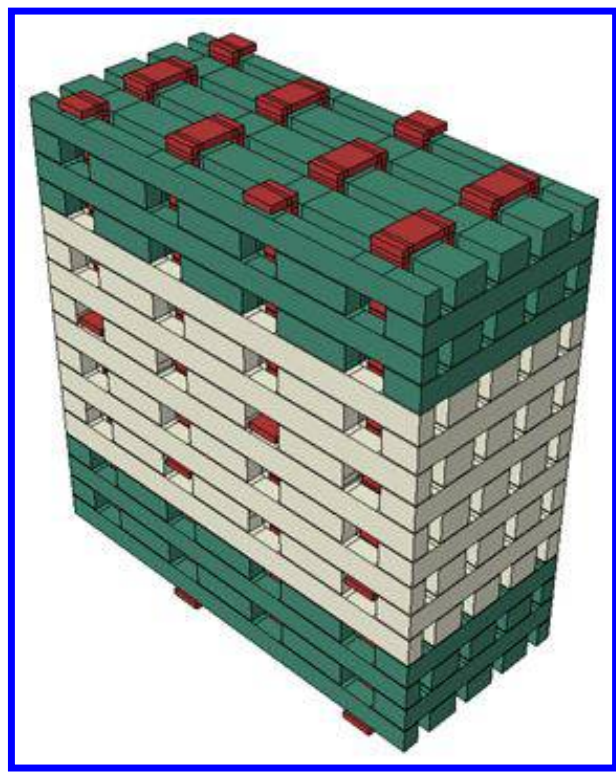

(c) Thick Symmetric

Figure 5. 3D CAD model of perfect Unit Cells 


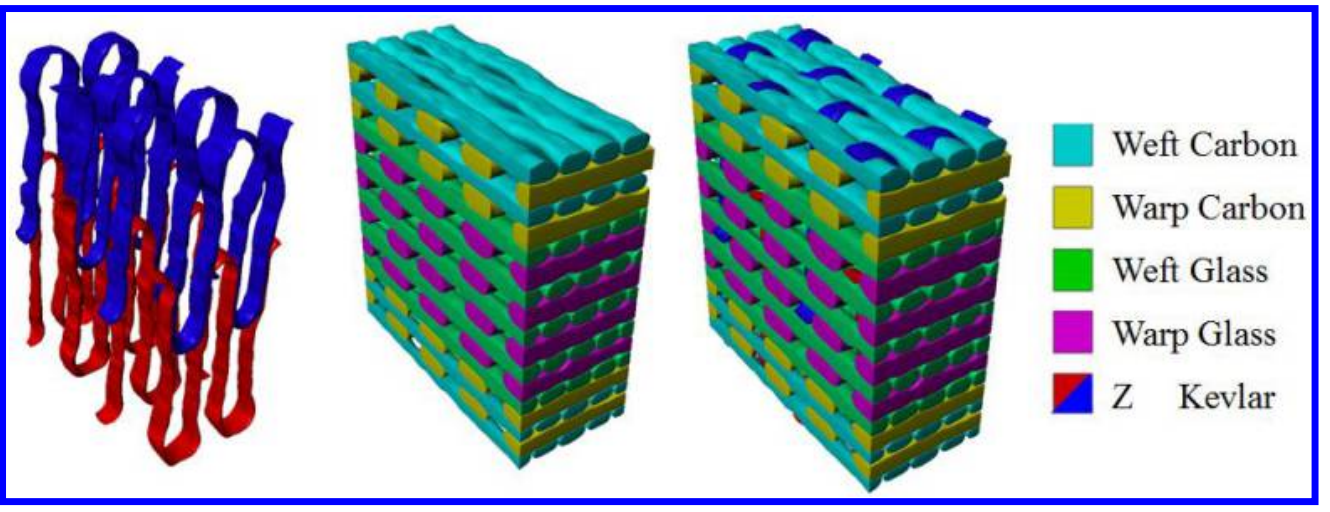

Figure 6. Segmentation of fiber tows using 'Simpleware'.

Different nonlinear responses have been reported for the matrix within a carbon tow than that in a glass tow. ${ }^{13}$ Table 5 summarizes the matrix nonlinear properties used in the two-scale micromechanics model for computing the response of each constituent tow. In addition, the pre-peak nonlinear properties of the surrounding matrix in the macro model is assumed to be the same as the one used for the glass tow.

Table 5. Matrix nonlinear properties used in this paper.

\begin{tabular}{cccc}
\hline & $\sigma_{y}(\mathrm{MPa})$ & $K_{1}(\mathrm{MPa})$ & $K_{2}$ \\
\hline Matrix (macroscale) & 25 & 1700 & 40 \\
In-situ carbon & 25 & 3500 & 60 \\
In-situ glass & 25 & 1700 & 40 \\
\hline
\end{tabular}

\section{Analysis Procedure}

The experimental results of the $\pm 45^{\circ}$ symmetric laminate tensile tests indicate that the matrix exhibits a nonlinear stress versus strain response due to the evolution of microdamage. In the proposed computational scheme, each fiber tow is homogenized as a 3D solid, therefore, micromechanical analysis must be implemented at the subscale to capture such nonlinear behavior.

\section{VII.A. Tow Pre-Peak Nonlinear Response: NCYL model}

Recently, Zhang and Waas ${ }^{1}$ developed a two-scale, micromechanics-based model for computing the nonlinear response of a unidirectional composite. In their model, a fiber-matrix concentric cylinder is used as the repeat unit cell (RUC), to represent a composite fiber tow. The two layers fiber-matrix analytical model ${ }^{1}$ has been extended to N-layer fiber-matrix cylinders ${ }^{2}$ to analyze the stress and strain fields for all constituent matrix layers. A representative N-layer concentric cylinder unit with fiber radius ' $a$ ' and matrix outer radius ' $b$ ' is shown in Figure 8 and the resulting fiber volume fraction is $V_{f}=a^{2} / b^{2}$.

Micromechanics is used to relate the globally applied composite strains to the fiber and matrix strains at the microscale through a six by six transformation matrix. A detailed description of the NCYL micromechanics model and its implementation in a multiscale analysis for progressive damage of textile composites is provided in the paper, ${ }^{2}$.

\section{VII.B. Tow Failure Mechanisms: Post-Peak Strain Softening Response}

Since the fiber tow pre-peak nonlinear response is attributed to matrix microdamage, no macroscopic damage criterion is required to drive the nonlinear damage progression. However, multiple catastrophic failure modes are observed in the experiment, including tensile failure due to fiber rupture and transverse and shear matrix failure in fiber tows which leads to tow splitting. These modes of failure result in a loss of load-carrying capability, followed by a post-peak strain softening response. Since the positive definiteness of the material tangent stiffness matrix is lost in the softening regime, the FE analysis will provide mesh dependent results if 


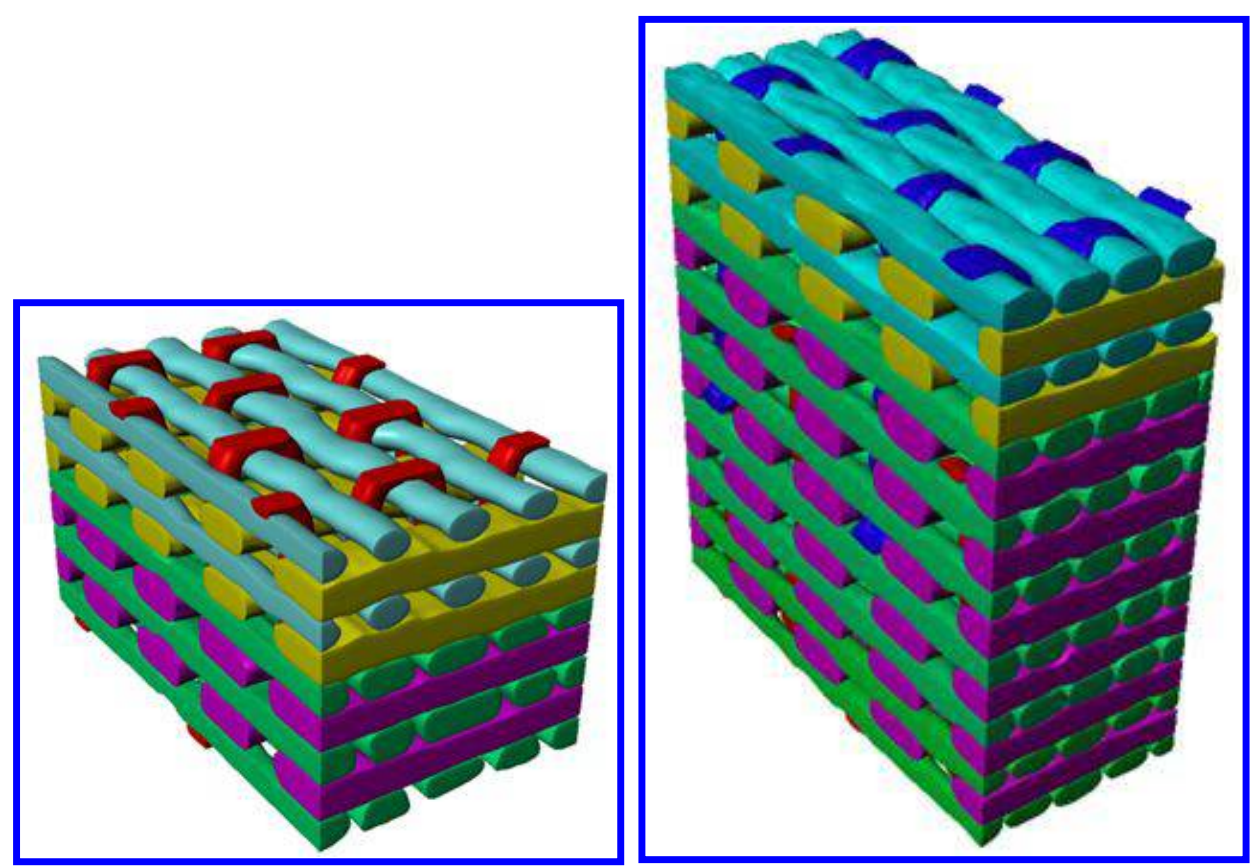

(a) Thin Asymmetric

(b) Thick Asymmetric

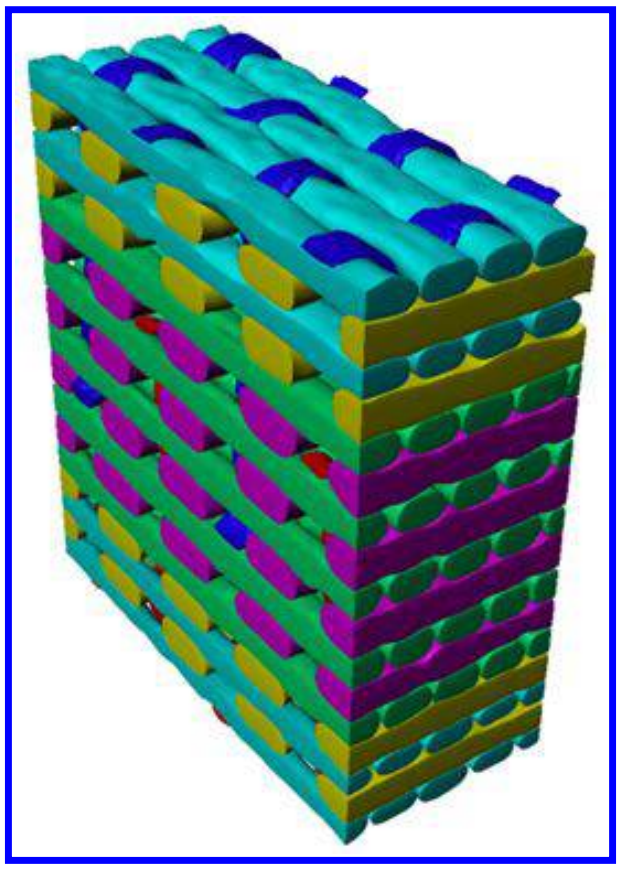

(c) Thick Symmetric

Figure 7. 3D CAD model of imperfect Unit Cells 


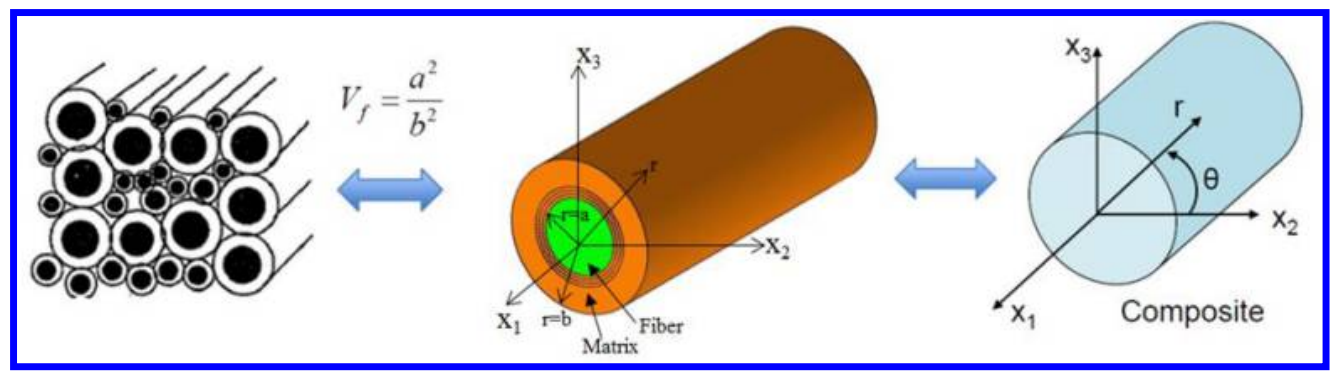

Figure 8. N-Layers Concentric Cylinder Model (NCYL).

no characteristic length associated with the softening response is introduced. As a result, the aforementioned 2-CYL model has to be supplemented by a suitable mesh objective approach for modeling the post-peak softening response at the macro-scale. There are a variety of theories and numerical implementations with respect to modeling of progressive damage and failure in composites. In this study, the the post-peak failure response at the macroscale is modeled using the smeared crack approach (SCA). This method was initially proposed by Rots et al. ${ }^{3}$ to model crack propagation and fracture in concrete. Extensions to modeling failure in composites are given in the papers,. ${ }^{4,10}$ The implementation provides mesh objectivity with respect to numerical discretization and uses standard 3D finite elements available in most commercial packages. This latter aspect is of great concern to practising engineers in industry who are concerned with validated computational methods.

A three dimensional SCA model for isotropic materials and a 2D orthotropic model are presented in Heinrich and Waas. ${ }^{4}$ The 3D isotropic SCA model is useful to describe the crack progression in the matrix material in a composite and the 2D orthotropic SCA model is used to model crack and damage progression within the layers (lamina) of a laminate. The 2D orthotropic SCA model is extended to a 3D orthotropic SCA model by Zhang ${ }^{11}$ and the failure response of fiber tows (post-peak softening response) is modeled using a new formulation, where the fiber tow is assumed to fail either in compression due to kink banding or in tension due to fiber breakage in the tows. It is further assumed that when the critical stress (either tension or compression) is reached, the crack plane aligns perpendicular to the fiber direction. Therefore, the crack orientation transformation matrix is determined by the material orientation rather than the state of stress.

In textile composites, fiber tows are surrounded by a polymer matrix, resulting in a complex state of stress. Due to the heterogeneity of the microstructure and the complexity of the stress fields, fiber tows exhibit multiple failure modes, including tow kinking in compression, tow breakage in tension, shear banding, and transverse and shear cracking. A unidirectional fiber tow is shown schematically in Figure 9, where fibers are aligned in 1-direction and the transverse 2-3 plane is assumed to be isotropic. Generally, the failure modes of a fiber tow can be grouped into two types, fiber failure modes that include tow breakage in tension and tow kinking in compression as shown in Figure 10 and matrix failure modes in which the failure plane is parallel to the fiber direction for transverse loading, as shown in Figure 11 for the 3 direction, which is equivalent to loading in the 2-direction due to assumed transverse isotropy. For matrix failure modes due to shear, the failure plane can be orthogonal to the fiber direction for the S12 and S13 cases, whereas the failure plane remains parallel to the fiber direction for the S21, S31, S23 and S32 cases, as shown in Figures 12, 13 and 14, respectively. This clearly shows that the strength S12 (S13) is higher than S21 (S31), as the shear failure orthogonal to the fiber direction which entails fiber shear failure, would result in a larger strength than the shear strength for failure parallel to the fiber direction. The assumption of matrix failure due to combined transverse and shear loading in each plane is similar to that proposed by Hashin. ${ }^{12}$ In the matrix failure modes, there exist three traction components on the crack plane, $\sigma_{n n}, \sigma_{n t}$ and $\sigma_{n l}$, which correspond to one normal traction and two shear tractions chosen appropriately from $\sigma_{22}, \sigma_{33}, \sigma_{23}, \sigma_{12}$ and $\sigma_{13}$ as shown in Figure 15. Thus the matrix failure modes account for cracks growing along the transverse normal, axial shear, and transverse shear directions, predicted based on the corresponding strengths and toughnesses of the composite.

It is evident from experiments that when a 3DWTC is subjected to tensile loading, the major failure modes for a fiber tow are tow tensile failure, matrix dominant failure modes such as axial shear cracking, and transverse failure. These modes have been observed within the warp/weft tows during deformation. The matrix dominant failure mode leads to a noticeable loss in load carrying capability, and therefore the 


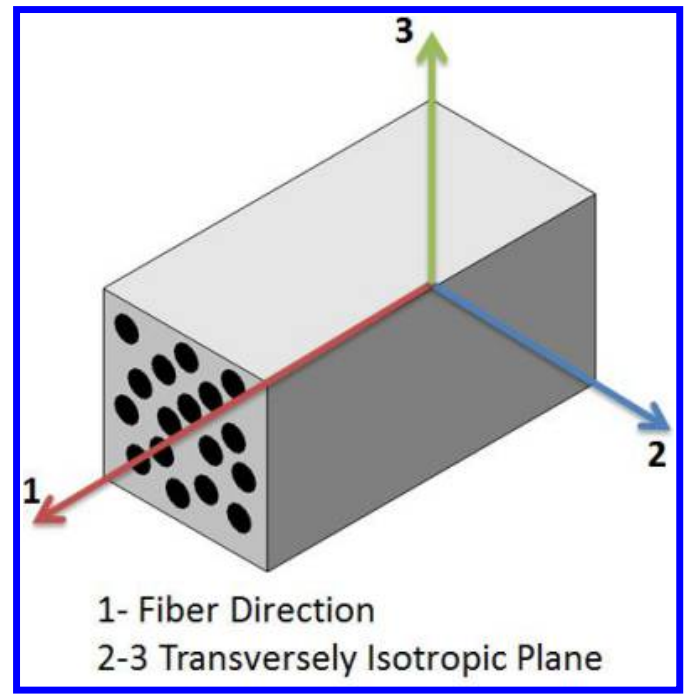

Figure 9. Unidirectional fiber tow (schematic).

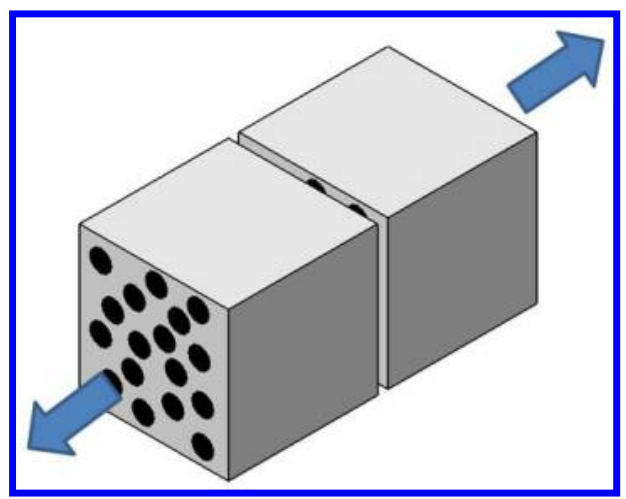

(a) Tow breakage (tension)

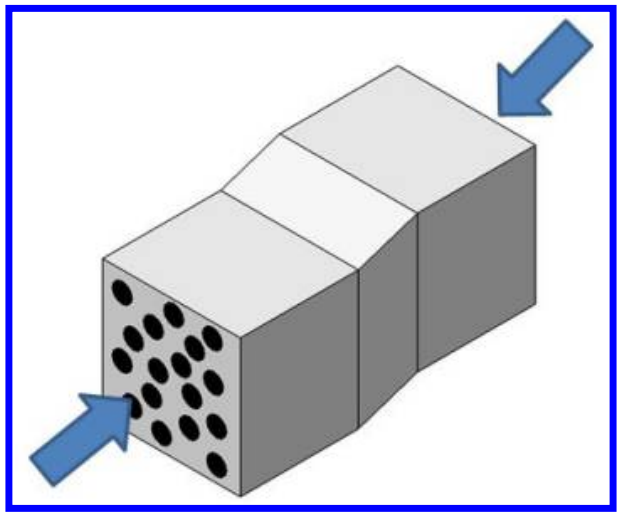

(b) Tow kinking (compression)

Figure 10. Fiber failure modes

resulting post-peak strain softening response must be included in the modeling. In this paper, all possible fiber and matrix failure modes in 1-2, 2-3 and 1-3 planes are introduced and implemented in the in-house developed 3D SCA code used for this research. The crack orientation transformation matrix, as determined by the material orientation for all three mutually perpendicular crack planes are provided in appendices A, $\mathrm{B}$ and $\mathrm{C}$, respectively. The failure properties, including the critical stress and fracture toughness for each mode of failure, are summarized in Table 6 for fiber tows and in Table 7 for SC-15 matrix respectively.

\section{Results and Discussion}

To obtain a full characterization of the composites nonlinear response and to extract the effective linear stiffness properties along with strength, two loading scenarios are considered, including tension in weft and warp directions for all three configurations.

\section{VIII.A. Stiffness and Strength comparison}

Effective stress-strain relations, based on applied load and cross sectional area of unit cells and global strains are determined from the simulations of all three configurations. The effective elastic moduli, strength and failure strains are extracted from these results for both perfect and imperfect unit cells. The elastic axial response show linearity in both weft and warp loading directions, followed by a slow gradual reduction of the tangent moduli and 'non-linear' behavior. The stress-strain response in the weft direction exhibits a 


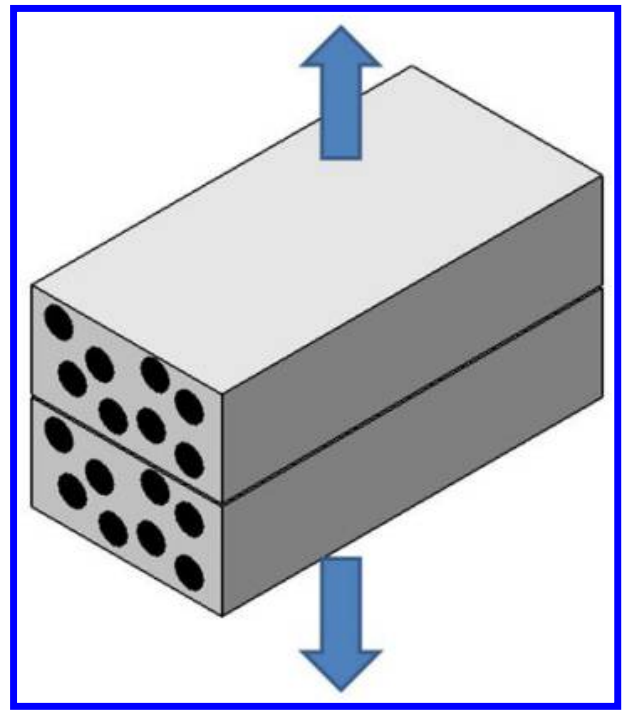

(a) Transverse tension (33)

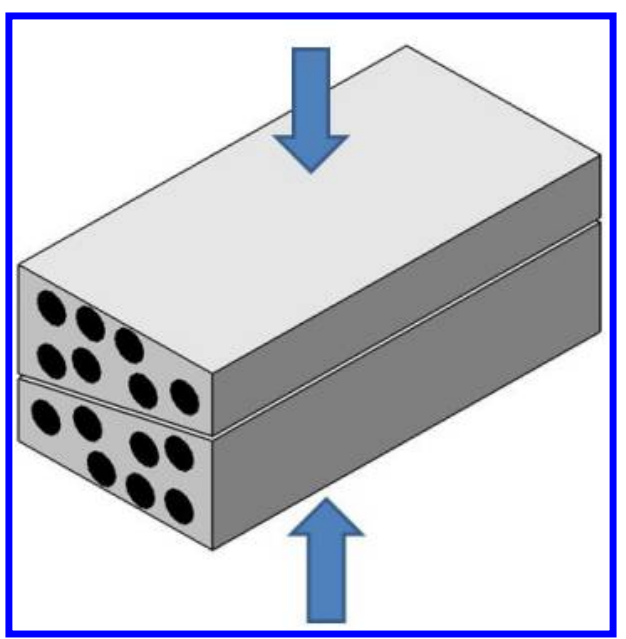

(b) Transverse compression (33)

Figure 11. Transverse failure modes in 3 direction

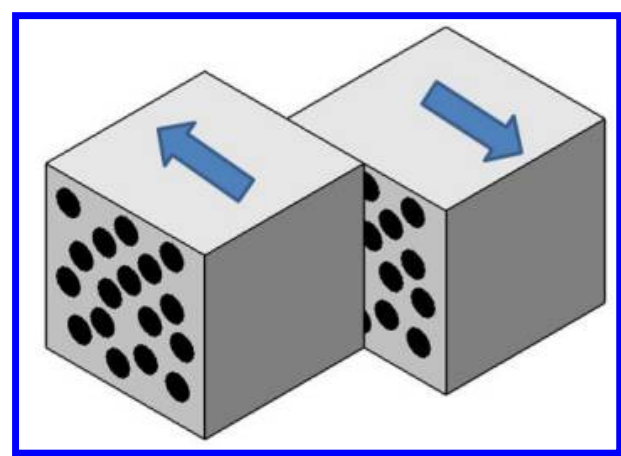

(a) Shear failure (S12)

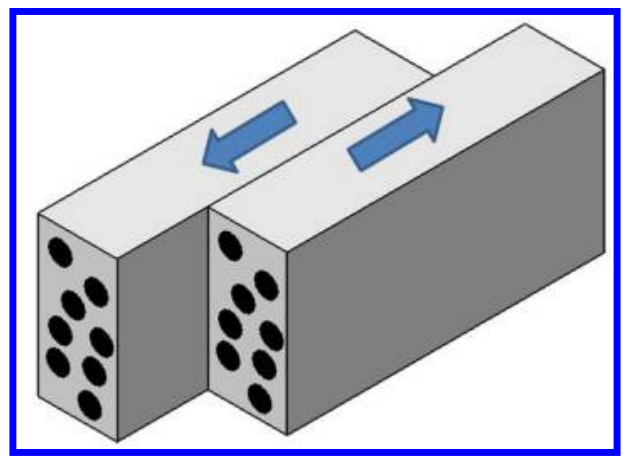

(b) Shear failure (S21)

Figure 12. Shear failure modes in 1-2 plane

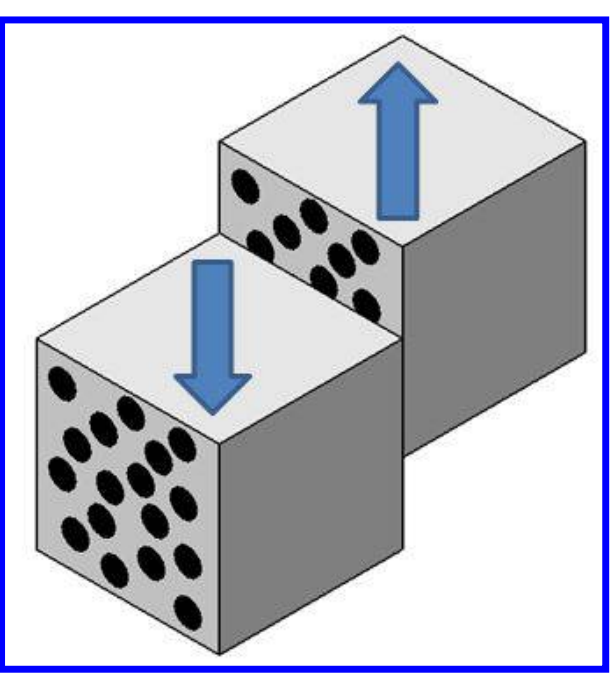

(a) Shear failure (S13)

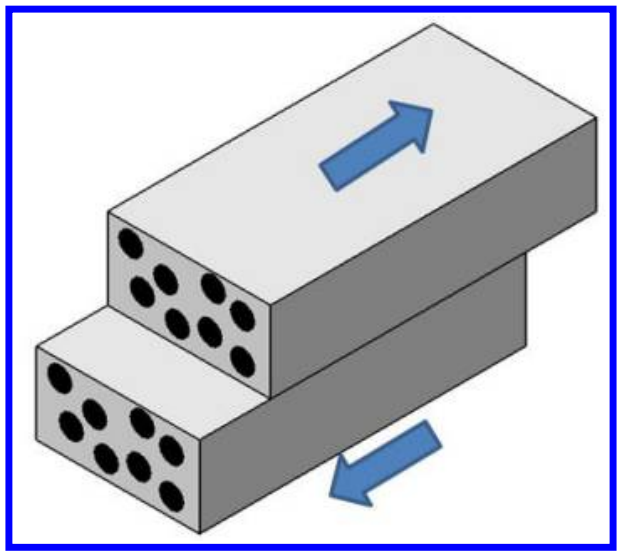

(b) Shear failure (S31)

Figure 13. Shear failure modes in 1-3 plane 


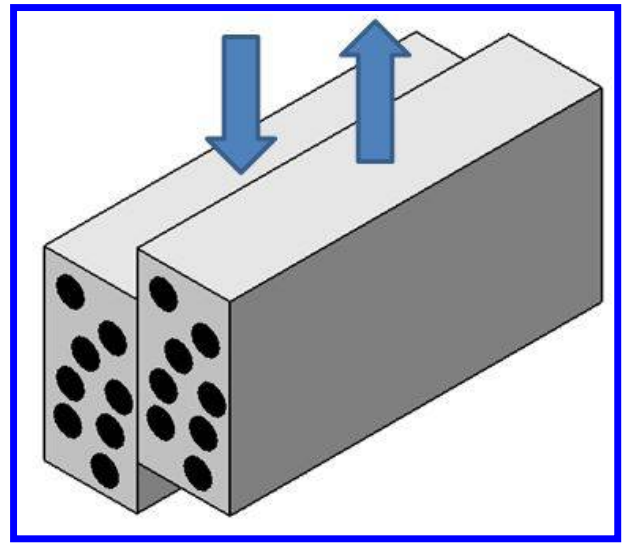

(a) Shear failure (S23)

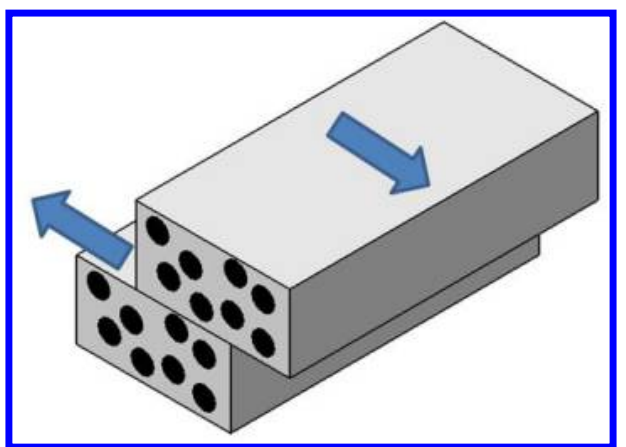

(b) Shear failure (S32)

Figure 14. Shear failure modes in 2-3 plane

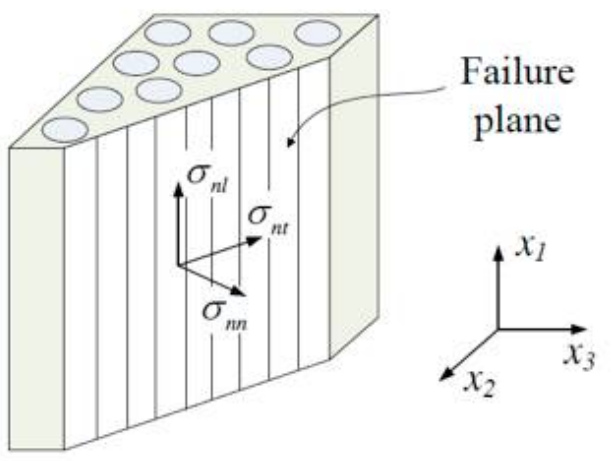

Figure 15. Matrix failure mode.

Table 6. Fiber tow failure properties used in the SCA model.

\begin{tabular}{lccc}
\hline Failure mode & Carbon & Glass & Kevlar \\
\hline $0^{\circ}$ tension $(\mathrm{MPa})$ & 3000 & 3700 & 3600 \\
$0^{\circ}$ compression $(\mathrm{MPa})$ & 977 & 720 & 720 \\
$90^{\circ}$ tension $(\mathrm{MPa})$ & 55 & 65 & 65 \\
$90^{\circ}$ compression $(\mathrm{MPa})$ & 247.3 & 247.3 & 247.3 \\
Shear $(\mathrm{MPa})$ & 37 & 35 & 35 \\
\hline$G_{I C F}^{+}(\mathrm{N} / \mathrm{mm})$ & 40 & 60 & 40 \\
$G_{I C F}^{-}(\mathrm{N} / \mathrm{mm})$ & 1 & 4 & 4 \\
$G_{I C M}^{+}(\mathrm{N} / \mathrm{mm})$ & 0.296 & 0.296 & 0.296 \\
$G_{I I C M}^{+}(\mathrm{N} / \mathrm{mm})$ & 0.647 & 0.647 & 0.647 \\
\hline
\end{tabular}

Table 7. SC-15 matrix failure properties used in the SCA model.

\begin{tabular}{lccc}
\hline Failure mode & $\sigma_{c r}(\mathrm{MPa})$ & $G_{I C M}^{+}(\mathrm{N} / \mathrm{mm})$ & $G_{I I C M}^{+}(\mathrm{N} / \mathrm{mm})$ \\
\hline Matrix tension & 30 & 0.296 & 0.647 \\
\hline
\end{tabular}

higher degree of linearity, whereas the warp direction shows more of a progression and early deviation from linearity due to large amount of matrix cracking, occurring in the thin matrix layers between the fiber tows. This phenomenon is attributed to the architectural influence and the orientation of Z-fibers with respect to 
weft and warp loading directions. The effective moduli for all three architectures are summarized in Table 8. Numerical predictions using the two-scale multiscale method showed excellent agreement with experimental data for in-plane elastic modulli in both weft and warp directions for the idealized perfect geometry without any imperfections. After including the in-situ geometrical imperfections and the fiber crookedness in the FE model, the predicted elastic moduli reduced with respect to the corresponding perfect models to some extent, depending on the amount of imperfections existing in different configurations. As shown, there is a reduction in stiffness after including the geometric imperfections. For these hybrid configurations, the weft direction is stiffer than the warp direction due to the fact that there is one additional glass fiber tow layer in the weft direction.

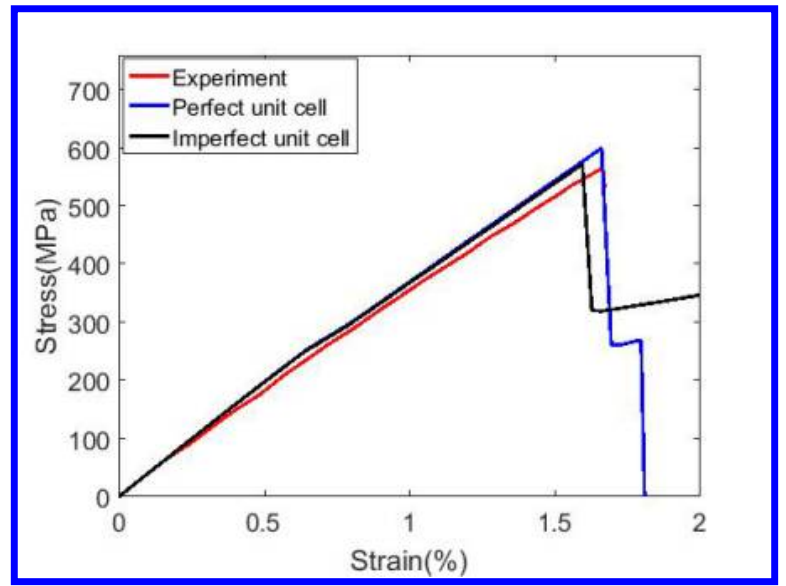

(a) Weft direction

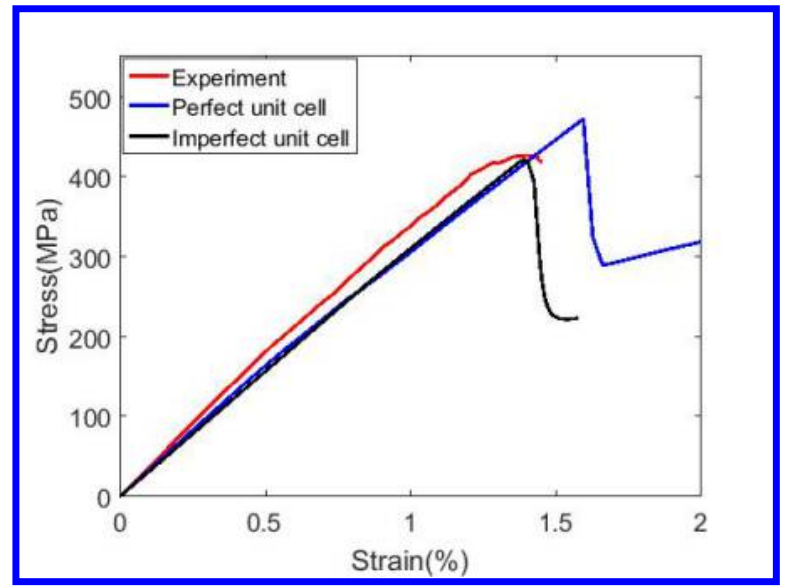

(b) Warp direction

Figure 16. Effective stress versus strain response for tensile loading in (a) Weft direction \& (b) Warp direction.

The weft and warp-direction tensile strength for all three architectures are summarized in Table 9 . The weft direction strengths are larger due to a higher fiber weight fraction compared to the warp direction. In imperfect unit cells, because of the imperfections in fiber alignment in both in-plane and thickness directions, there is significant fiber tow splitting observed in transverse and Z-fiber tows. The predicted tensile strength for imperfect models reduced the respective perfect model values in all the configurations and predictions are closer to experimental values. The global response of both perfect and imperfect unit cells for Thin Asymmetric configuration is shown in Figure 16 as a demonstration, which shows the effect of including manufacturing induced imperfections in the progressive damage and failure analysis of these textile composites.

Table 8. Stiffness comparison between Experiment and Simulation results

\begin{tabular}{ccccc}
\hline Architecture & EffectiveTensileModulus & $\begin{array}{c}\text { Experiment } \\
(\mathrm{GPa})\end{array}$ & $\begin{array}{c}\text { Simulation(Perfect) } \\
(\mathrm{GPa})\end{array}$ & $\begin{array}{c}\text { Simulation(Imperfect) } \\
(\mathrm{GPa})\end{array}$ \\
\hline Thin Asymmetric & Warp & $36.497 \pm 11.34 \%$ & 34.57 & 34.43 \\
& Weft & $39.612 \pm 3.58 \%$ & 39.75 & 39.6 \\
Thick Asymmetric & Warp & $29.1 \pm 13.78 \%$ & 28.43 & 28.17 \\
& Weft & $57.185 \pm 5.47 \%$ & 55.85 & 34.95 \\
Thick Symmetric & Warp & $37.023 \pm 11.34 \%$ & 34.87 & 39.12 \\
& Weft & $41.775 \pm 3.58 \%$ & 40.85 & 35 \\
\hline
\end{tabular}

\section{VIII.B. Progressive damage during quasi-static tensile loading}

The multiscale methodology described earlier combines pre-peak nonlinearity using the NCYL secant stiffness method and the post-peak strain softening response using the smeared crack approach (SCA). The combined models are used together to conduct a thorough investigation of the damage and failure mechanisms in the $3 \mathrm{D}$ hybrid textile composite of all three configurations discussed above. The progressive damage and failure 
Table 9. Strength comparison between Experiment and Simulation results

\begin{tabular}{ccccc}
\hline Architecture & Ultimatestrength & $\begin{array}{c}\text { Experiment } \\
(\mathrm{MPa})\end{array}$ & $\begin{array}{c}\text { Simulation(Perfectmodel) } \\
(\mathrm{MPa})\end{array}$ & $\begin{array}{c}\text { Simulation(Imperfectmodel) } \\
(\mathrm{MPa})\end{array}$ \\
\hline \multirow{2}{*}{ Thin Asymmetric } & Warp & 427.4 & 491.3 & 410.4 \\
& Weft & 566.84 & 600.5 & 572.6 \\
\multirow{2}{*}{ Thick Asymmetric } & Warp & 346.65 & 380.67 & 322.81 \\
& Weft & 510.64 & 467.6 & 461.3 \\
\multirow{2}{*}{ Thick Symmetric } & Warp & 393.32 & 446.81 & 403 \\
& Weft & 469.33 & 467.61 & 449.7 \\
\hline
\end{tabular}

response of Thin Asymmetric configuration, in both weft and warp directions subjected to uniaxial quasistatic tensile tests are shown in Figure 17 to Figure 20 for perfect unit cells without geometrical imperfections. In these figures, the progressive failure status at different percentages of the failure strength are shown as contour plots of progressive failure flags of the elements.

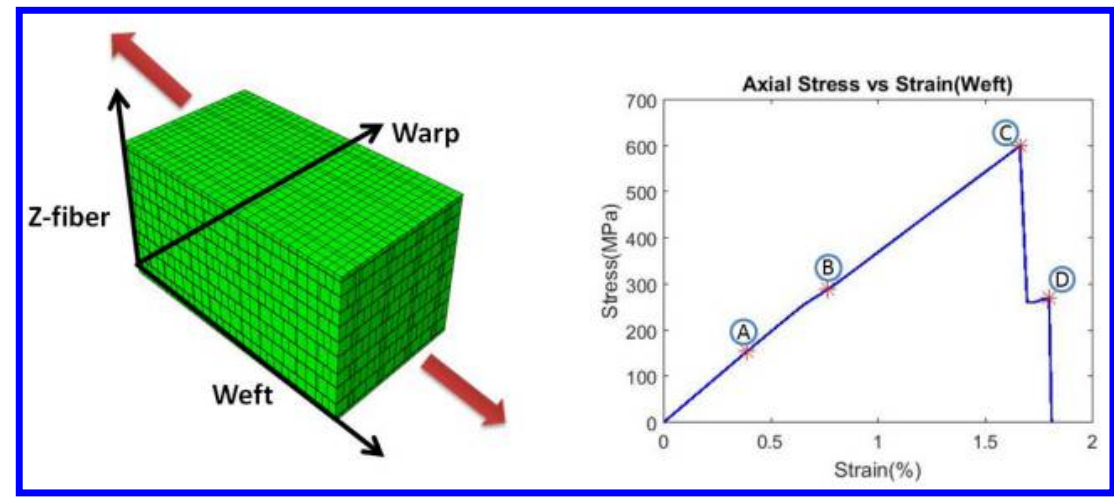

Figure 17. Effective stress versus strain response for tensile loading in weft direction

The damage in these composites starts near the location of Z-fiber tows and at the edges of the in-plane fiber tows oriented transversely to the loading direction. Due to this reason, there is significant amount of local disbonds and matrix cracking occurring in the case of warp direction loading compared to weft direction, as shown in figures 18 and 20. This failure event leads to development of transverse cracks within the in-plane and vertical tows (referred to as 'tow splitting') during the progressive loading process; these are followed by fiber tow breakage in the axial direction at a very late stage of loading and finally causes the 'two-piece' failure.

\section{VIII.C. Effect of hybridization}

The tensile test simulations are carried out for both weft and warp directions and the stress versus strain responses are obtained as shown in Figure 21. These simulation results are compared with the experimental results reported in,. ${ }^{6}$ Hybrid 3DWTC of three different architectures are examined and the effect of hybridization is investigated to understand the effective stiffness and strength in tension. Thin Asymmetric configuration shows the highest strength in both warp and weft directions. Additional layers of carbon decreased the overall strength due to change in load transfer path to the fiber tows. The architectures and orientations of fiber tows have a strong impact on the localization of strains in the thin matrix layers between fiber tows, which cause matrix micro-cracking and initiates the failure event. Hybridization, by adding carbon to an existing glass fiber system, appears to provide change in tensile stiffness but at the expense of a lower ultimate tensile strength.

\section{Conclusions}

In this work, three different configurations of hybridized 3DWTC are simulated for uniaxial tensile response to determine the effect of hybridization and compute the effective stiffness and strength in tension 


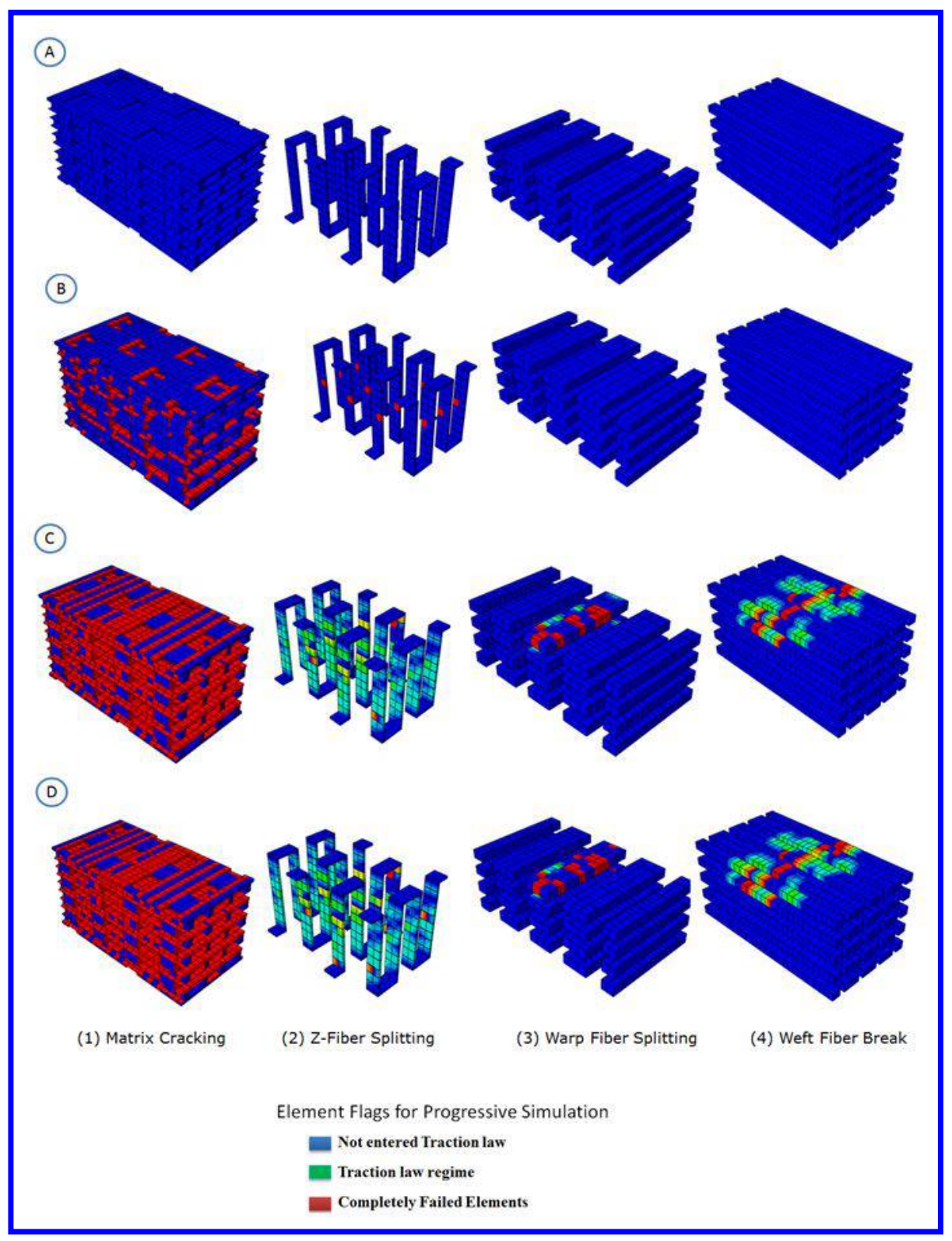

Figure 18. Progressive failure for tensile loading in weft direction

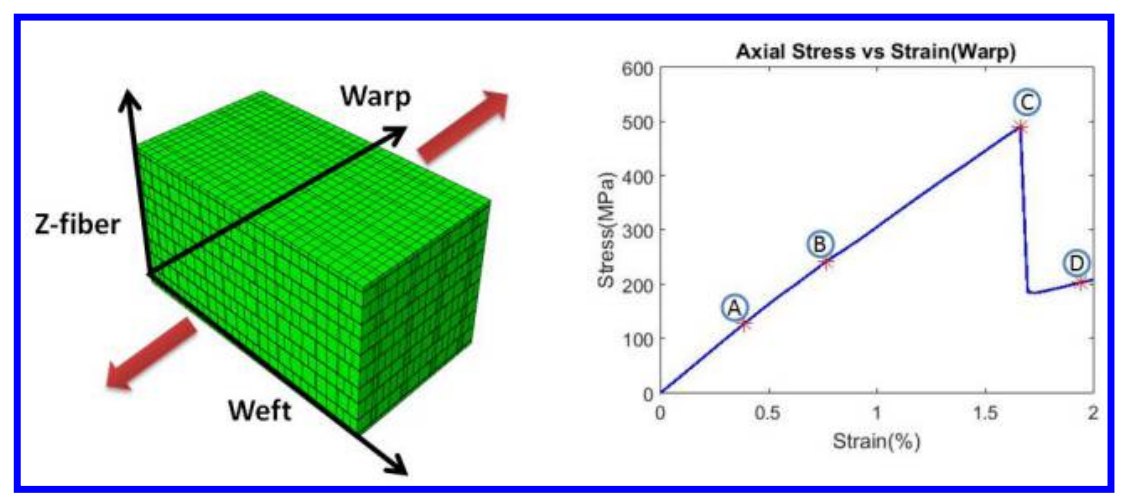

Figure 19. Effective stress versus strain response for tensile loading in warp direction 


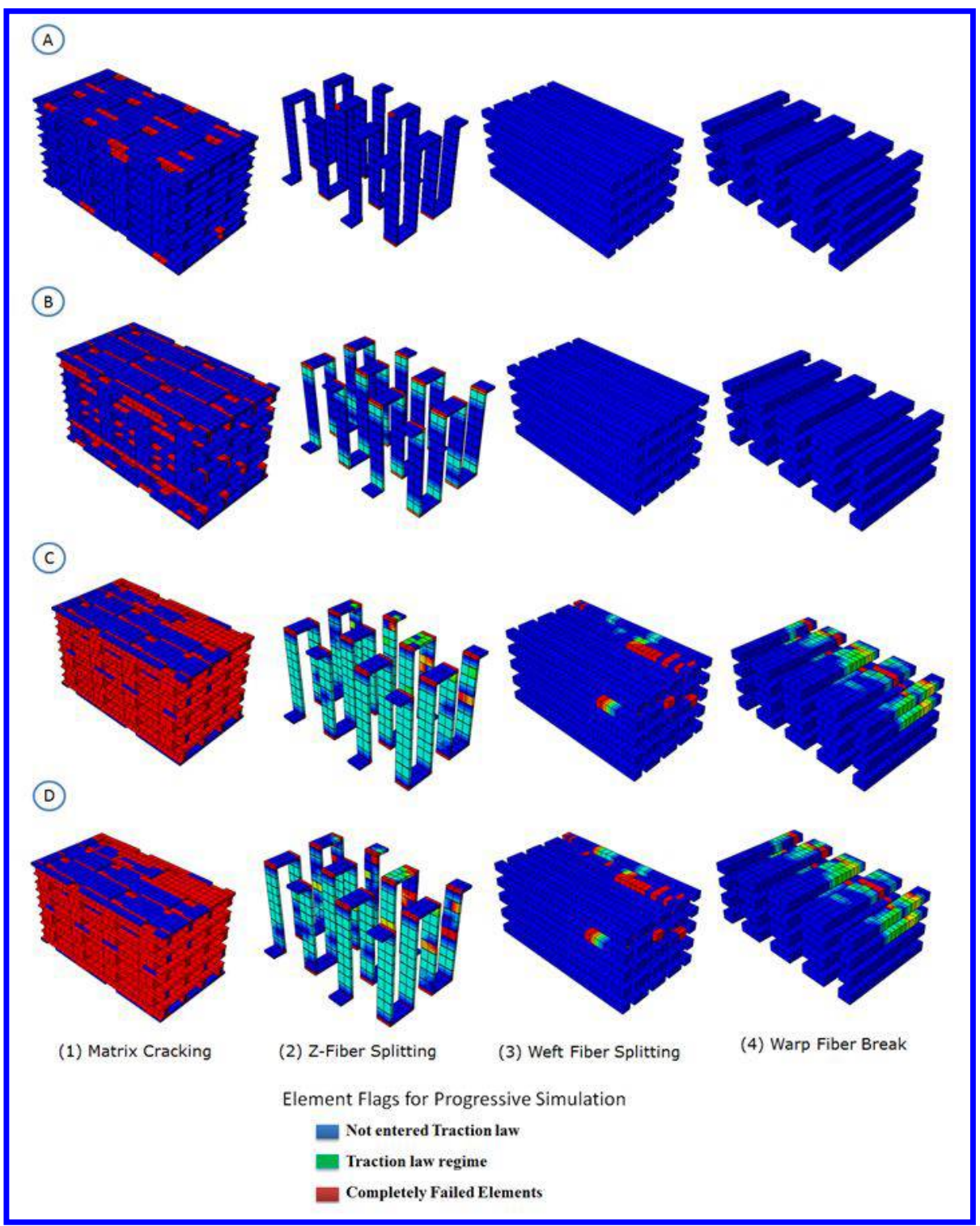

Figure 20. Progressive failure for tensile loading in warp direction

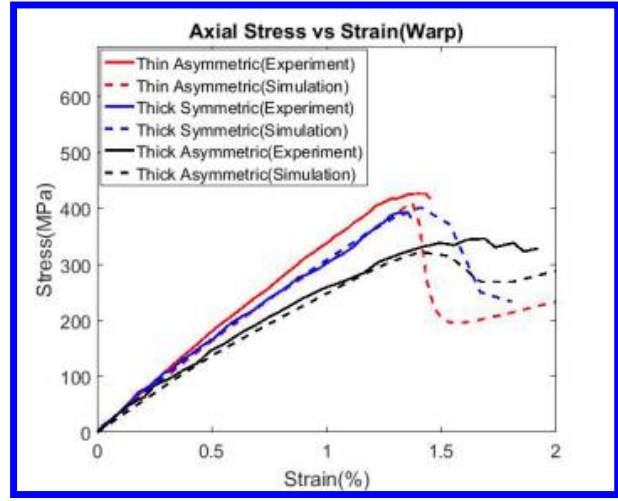

(a) Tension in warp direction

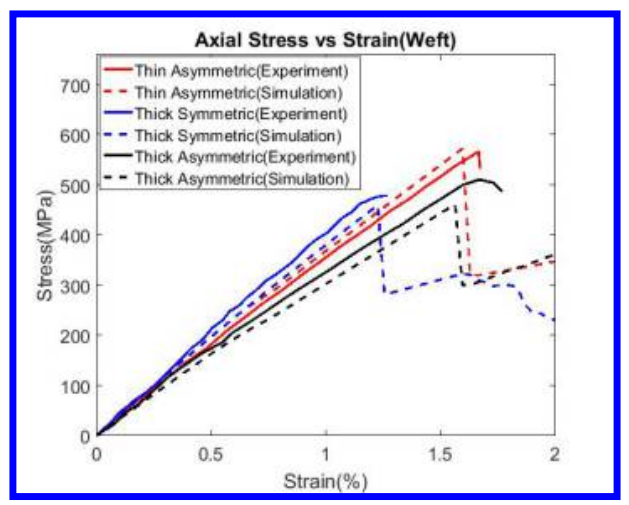

(b) Tension in weft direction

Figure 21. Comparison of Stress vs. Strain responses for the different 3DWTCs. 
for both warp and weft directions. A Micro-CT analysis is carried out to characterize in-situ microstructural geometric imperfections. This Micro-CT analysis helps to extract essential inputs like dimensions of Unit Cell, cross-sectional details of fiber tows in both warp and weft directions and volume fraction of pores. Based on these inputs, an idealized 3D CAD model of a Unit Cell is constructed. Furthermore, the micro CT data is used in conjunction with a software tool, 'Simpleware', to generate a FE mesh that captures in-situ geometry, and providing a platform to carry out a 2 scale analysis on realistic imperfect unit cells. A subscale micromechanics 2CYL model, with an analytical solution at the sub-scale is used to establish a computational framework to predict the effective nonlinear response of 3DWTCs. The influence of matrix microdamage at the microscale manifests as the degradation of the effective fiber tow stiffness at the macroscale through a secant moduli approach. Since, fully analytical solutions are utilized for the subscale micromechanics analysis, the proposed method offers a lower computational cost and is suitable for large scale progressive damage and failure analysis of textile composite structures. The linear stiffness and strength of all the textile architectures studied are predicted well when compared to experimental results. The approach presented here can be used to understand and quantify the effects of hybridization and textile architecture on the tensile response of textile composites, in terms of constituent properties. Furthermore, the modeling framework can be used to quantify the effects of uncertain constituent properties on the overall response of textile composites, an essential task in an ICME (integrated computational materials engineering) of composites.

\section{Appendix}

\section{A. Calculation of crack orientation transformation matrix for 1-1 plane (fiber failure mode)}

Assuming that the global coordinates are represented by the $1-2-3$ triad while the local crack orientation is denoted by the $1^{\prime}-2^{\prime}-3^{\prime}$ triad, with the crack normal aligned with the $1^{\prime}$-direction, it follows that,

$$
\left\{\begin{array}{l}
\epsilon_{11} \\
\epsilon_{22} \\
\epsilon_{33} \\
\gamma_{12} \\
\gamma_{13} \\
\gamma_{23}
\end{array}\right\}=[\boldsymbol{N}]\left\{\begin{array}{l}
\epsilon_{11}^{\prime} \\
\gamma_{12}^{\prime} \\
\gamma_{13}^{\prime}
\end{array}\right\}
$$

where,

$$
\boldsymbol{N}=\left[\begin{array}{ccc}
a_{11}^{2} & a_{11} a_{21} & a_{11} a_{31} \\
a_{12}^{2} & a_{12} a_{22} & a_{12} a_{32} \\
a_{13}^{2} & a_{13} a_{23} & a_{13} a_{33} \\
2 a_{11} a_{12} & a_{11} a_{22}+a_{12} a_{21} & a_{11} a_{32}+a_{12} a_{31} \\
2 a_{11} a_{13} & a_{11} a_{23}+a_{13} a_{21} & a_{11} a_{33}+a_{13} a_{31} \\
2 a_{12} a_{13} & a_{12} a_{23}+a_{13} a_{22} & a_{12} a_{33}+a_{13} a_{32}
\end{array}\right]
$$

and $a_{i j}^{\prime} s$ are the direction cosines governing the space vector transformation as,

$$
x_{i}^{\prime}=a_{i p} x_{p}
$$

When the SCA is implemented for a fiber tow failure due to tension or compression, the crack plane is assumed to be perpendicular to the fiber direction. Thus, the $1^{\prime}$-axis that defines the crack normal coincides with the 1-axis that denotes the fiber direction, and $\boldsymbol{N}$ is reduced to, 


$$
\boldsymbol{N}=\left[\begin{array}{lll}
1 & 0 & 0 \\
0 & 0 & 0 \\
0 & 0 & 0 \\
0 & 1 & 0 \\
0 & 0 & 1 \\
0 & 0 & 0
\end{array}\right]
$$

\section{B. Calculation of crack orientation transformation matrix for 2-2 plane (matrix failure mode)}

Assuming that the global coordinates are represented by the $1-2-3$ triad while the local crack orientation is denoted by the $1^{\prime}-2^{\prime}-3^{\prime}$ triad, with the crack normal aligned with the $1^{\prime}$-direction, it follows that,

$$
\left\{\begin{array}{l}
\epsilon_{11} \\
\epsilon_{22} \\
\epsilon_{33} \\
\gamma_{12} \\
\gamma_{13} \\
\gamma_{23}
\end{array}\right\}=[\boldsymbol{N}]\left\{\begin{array}{l}
\epsilon_{11}^{\prime} \\
\gamma_{12}^{\prime} \\
\gamma_{13}^{\prime}
\end{array}\right\}
$$

where,

$$
\boldsymbol{N}=\left[\begin{array}{ccc}
a_{11}^{2} & a_{11} a_{21} & a_{11} a_{31} \\
a_{12}^{2} & a_{12} a_{22} & a_{12} a_{32} \\
a_{13}^{2} & a_{13} a_{23} & a_{13} a_{33} \\
2 a_{11} a_{12} & a_{11} a_{22}+a_{12} a_{21} & a_{11} a_{32}+a_{12} a_{31} \\
2 a_{11} a_{13} & a_{11} a_{23}+a_{13} a_{21} & a_{11} a_{33}+a_{13} a_{31} \\
2 a_{12} a_{13} & a_{12} a_{23}+a_{13} a_{22} & a_{12} a_{33}+a_{13} a_{32}
\end{array}\right]
$$

and $a_{i j}^{\prime} s$ are the direction cosines governing the space vector transformation as,

$$
x_{i}^{\prime}=a_{i p} x_{p}
$$

When the SCA is implemented for a fiber tow split in 2-direction due to transverse loading, the crack plane is assumed to be parallel to the fiber direction. Thus, the $1^{\prime}$-axis that defines the crack normal is perpendicular to the 1-axis that denotes the fiber direction, and $N$ is reduced to,

$$
\boldsymbol{N}=\left[\begin{array}{lll}
0 & 0 & 0 \\
0 & 0 & 0 \\
1 & 0 & 0 \\
0 & 0 & 0 \\
0 & 0 & 1 \\
0 & 1 & 0
\end{array}\right]
$$

\section{Calculation of crack orientation transformation matrix for 3-3 plane (matrix failure mode)}

Assuming that the global coordinates are represented by the $1-2-3$ triad while the local crack orientation is denoted by the $1^{\prime}-2^{\prime}-3^{\prime}$ triad, with the crack normal aligned with the $1^{\prime}$-direction, it follows that, 


$$
\left\{\begin{array}{l}
\epsilon_{11} \\
\epsilon_{22} \\
\epsilon_{33} \\
\gamma_{12} \\
\gamma_{13} \\
\gamma_{23}
\end{array}\right\}=[\boldsymbol{N}]\left\{\begin{array}{l}
\epsilon_{11}^{\prime} \\
\gamma_{12}^{\prime} \\
\gamma_{13}^{\prime}
\end{array}\right\}
$$

where,

$$
\boldsymbol{N}=\left[\begin{array}{ccc}
a_{11}^{2} & a_{11} a_{21} & a_{11} a_{31} \\
a_{12}^{2} & a_{12} a_{22} & a_{12} a_{32} \\
a_{13}^{2} & a_{13} a_{23} & a_{13} a_{33} \\
2 a_{11} a_{12} & a_{11} a_{22}+a_{12} a_{21} & a_{11} a_{32}+a_{12} a_{31} \\
2 a_{11} a_{13} & a_{11} a_{23}+a_{13} a_{21} & a_{11} a_{33}+a_{13} a_{31} \\
2 a_{12} a_{13} & a_{12} a_{23}+a_{13} a_{22} & a_{12} a_{33}+a_{13} a_{32}
\end{array}\right]
$$

and $a_{i j}^{\prime} s$ are the direction cosines governing the space vector transformation as,

$$
x_{i}^{\prime}=a_{i p} x_{p}
$$

When the SCA is implemented for a fiber tow split in 3-direction due to transverse loading, the crack plane is assumed to be parallel to the fiber direction. Thus, the $1^{\prime}$-axis that defines the crack normal is perpendicular to the 1-axis that denotes the fiber direction, and $\boldsymbol{N}$ is reduced to,

$$
\boldsymbol{N}=\left[\begin{array}{lll}
0 & 0 & 0 \\
1 & 0 & 0 \\
0 & 0 & 0 \\
0 & 0 & 1 \\
0 & 0 & 0 \\
0 & 1 & 0
\end{array}\right]
$$

\section{Acknowledgments}

The authors are grateful for financial sponsorship from the Army Research Office (Drs. Larry Russel and Asher Rubinstein, as Program Managers) and the encouragement and support of Dr. Chian Yen at the Army Research Laboratory, Aberdeen Proving Ground, MD. We gratefully acknowledge the HYAK and FLUX high-performance computing clusters at the University of Washington and University of Michigan, respectively, for enabling this study.

\section{References}

12 Zhang, D. and Waas, A.M., A micromechanics based multiscale model for nonlinear composites, Acta Mechanica, Vol. 225, No. 4-5, 2014, pp. 1391-1417. doi:10.1007/s00707-013-1057-1.

${ }^{2}$ Patel, D.K., Hasanyan, A.D. and Waas, A.M., N-Layer concentric cylinder model (NCYL): an extended micromechanicsbased multiscale model for nonlinear composites, Acta Mechanica. doi:10.1007/s00707-016-1696-0.

${ }^{3}$ Rots, G., Nauta, P., Kusters, G. M. A. and Blaauwendraad, J., Smeared crack approach and fracture localization in concrete, HERON , Vol. 30, No. 1, 1985, pp. 1-48.

${ }^{4}$ Heinrich, C. and Waas, A.M., Investigation of progressive damage and fracture in laminated composites using the smeared crack approach, 53rd AIAA/ASME/ASCE/AHS/ASC Structures, Structural Dynamics and Materials Conference, Honolulu, Hawaii, April 2012, AIAA 2012-1537.

${ }^{5}$ Patel, D.K. and Waas, A.M., Damage and failure modelling of hybrid three-dimensional textile composites: a mesh objective multi-scale approach, Philosophical Transactions of The Royal Society A, Vol. 374, Issue $2071,2016$. doi:10.1098/rsta.2016.0036.

${ }^{6}$ Pankow M., Yen C.F., Rudolph M., Justusson B., Zhang D. and Waas A.M., Experimental Investigation on the Deformation Response of Hybrid 3D Woven Composites, AIAA SciTech, 53rd AIAA/ASME/ASCE/AHS/ASC Structures, Structural Dynamics and Materials Conference, Honolulu, Hawaii, April 2012, AIAA 2012-1572. 
${ }^{7}$ Herakovich, C.T., Mechanics of Fibrous Composites, John Wiley and Sons, Inc., 1998.

${ }^{8}$ Aboudi, J., Arnold, S.M. and Bednarcyk, B.A., Micromechanics of Composite Materials: A Generalized Multiscale Analysis Approach, Elsevier, 1st ed., 2013.

${ }^{9} \mathrm{Ng}$, W. H., Salvi, A.G., and Waas, A. M., Characterization of the in-situ non-linear shear response of laminated fiber-reinforced composites," Composites Science and Technology, Vol. 70, No. 7, July 2010, pp. 1126-1134. doi:10.1016/j.compscitech.2010.02.024.

${ }^{10}$ Zhang, D., Waas, A. M. and Yen, C., Progressive failure analysis on textile composites, AIAA SciTech, 55rd AIAA/ASME/ASCE/AHS/ASC Structures, Structural Dynamics and Materials Conference, National Harbor, Maryland, January 2014, AIAA 2014-0157.

${ }^{11}$ Zhang, D., Progressive Damage and Failure Analysis of 3D Textile Composites Subjected to Flexural Loading, Ph.D. thesis, University of Michigan, 2014.

${ }^{12}$ Hashin, Z., Failure Criteria for Unidirectional Fiber Composites," Journal of Applied Mechanics, Vol. 47, No. 2, 1980, pp. 329. doi:10.1115/1.3153664.

13 Zhang, D., Waas, A.M. and Yen, C.F., "Progressive damage and failure response of hybrid 3D textile composites subjected to flexural loading," Part I: Experimental studies. Int. J. Solids Struct., Vol. 75-76, pp. 309-320. doi:10.1016/j.ijsolstr.2015.06.034. 